\title{
Endocarditis infecciosa por Abiotrophia defectiva diagnosticada por espectrometría de masas (MALDI-TOF MS) en Argentina
}

\author{
Flavio Lipari, Matías Martínez, Daniela Hernández, María Laborie y Juan P. Caeiro
}

Hospital Privado Universitario de Córdoba. Servicio de Infectología (FL, JPC) Servicio de Cardiología (MM) Servicio de Microbiología (DH) Servicio de Anatomía Patológica

(MVL)

Sin conflictos de intereses. Sin fuentes de financiación.

Recibido: 1 de abril de 2016 Aceptado: 21 de octubre de 2016

Correspondencia a: Flavio Lipari fglipari@gmail.com

\section{Infective endocarditis by Abiotrophia defectiva diagnosed by mass spectrometry (MALDI-TOF MS) in Argentina}

We report the case of a 63-year-old woman with congestive heart failure due to a bicuspid aortic valve and severe aortic stenosis. The patient had a febrile syndrome with positive blood cultures for Abiotrophia defectiva, Transesophageal echocardiogram revealed the presence of paravalvular abscess, which was treated by a successful valve replacement. The patient received appropriate antibiotic therapy with intravenous vancomycin, leading to a successful response. The use of MALDI-TOF MS as a rapid and specific method for the microbiological diagnosis is discussed in the following report.

Key words: Endocarditis, abscess, Abiotrophia defectiva. MALDI-TOF MS.

Palabras clave: Endocarditis, absceso, Abiotrophia defectiva, MALDI-TOF MS.

\section{Introducción}

L os géneros Abiotrophia y Granulicatella, previamente denominados variantes nutricionales de Streptococcus (VNS) se desarrollan en medios líquidos, con la presencia de clorhidrato de piridoxal y clorhidrato de cisteína ${ }^{1}$. Estas bacterias de crecimiento dificultoso se ven como satélites de colonias de otras bacterias (Staphylococus aureus) en medios sólidos comunes (satelitismo) $)^{2}$. Abiotrophia spp. es un microorganismo comensal de la mucosa oral, intestinal y genital ${ }^{3}$.

La infección más frecuentemente documentada al género Abiotrophia ha sido la endocarditis ${ }^{3}$, causando aproximadamente $5 \%$ de los casos ${ }^{4}$.

MALDI-TOF MS (Matrix Assisted Laser Desorption Ionization Time of Flight. Mass Spectrometry) es un método microbiológico moderno y efectivo para la identificación rápida de microorganismos ${ }^{5}$. Las especies de Granulicatella y Abiotrophia pueden ser diferenciadas por medio de esta técnica en forma precoz y segura ${ }^{6}$.

Son escasos los reportes de endocarditis infecciosa por este microorganismo en Latinoamérica ${ }^{7}$, y ninguno con la utilización de MALDI-TOF MS.

\section{Caso clínico}

Mujer de 63 años de edad con antecedentes de hipotiroidismo (post-hemitiroidectomía derecha por enfermedad de Plummer), hipoparatiroidismo primario (tiroidectomía total y paratiroidectomía), osteoporosis, fumadora (20 cigarrillos al día) y válvula aórtica bicúspide con estenosis aórtica crítica diagnosticada hacía cinco años aproximadamente.

Consultó por disnea clase funcional III (clasificación funcional de New York Heart Association), agravada por episodios de disnea paroxística nocturna de dos semanas de evolución, progresión de edema en extremidades inferiores y fiebre no cuantificada. Al examen físico presentaba taquipnea, con saturación de oxigeno $94 \%$ con aire ambiental, frecuencia cardíaca 98 latidos por min, y presión arterial de 110/70 $\mathrm{mmHg}$. Al examen pulmonar presentaba crépitos hasta un tercio de ambos campos pulmonares, ingurgitación yugular y edema de extremidades inferiores. Al examen cardíaco: ruidos cardíacos normales, con un soplo sistólico aórtico de intensidad 3/6.

El laboratorio de ingreso mostró una hiponatremia (130 $\mathrm{mmol} / \mathrm{l}$ ), potasio y cloro normales, recuento de leucocitos 9.500 células $/ \mathrm{mm}^{3}$, neutrófilos $91 \%$, hemoglobina 10,8 $\mathrm{g} / \mathrm{dl}$, una proteína $\mathrm{C}$ reactiva de $7,34 \mathrm{mg} / \mathrm{dl}$ y un péptido natriurético cerebral: $2.885 \mathrm{pg} / \mathrm{ml}$.

Se internó en la unidad coronaria por una insuficiencia cardíaca descompensada. Se inició tratamiento con vasodilatadores y diuréticos con buena respuesta inicial. Se realizó una cinecoronariografía que mostró arterias coronarias sin lesiones significativas. Durante su primer día de internación presentó fiebre de $38,5^{\circ} \mathrm{C}$, sin descompensación hemodinámica. Se tomaron dos hemocultivos y urocultivo y se inició tratamiento con antimicrobianos (piperacilina-tazobactam 4,5 g, fraccionado cada ocho horas y vancomicina $1 \mathrm{~g}$, cada $12 \mathrm{~h}$ ), además de una infusión continua intravenosa de milrinona y furosemida. 


\section{Caso Clínico}

A las $48 \mathrm{~h}$, se informaron los hemocultivos positivos para cocobacilos grampositivos. Posteriormente, la colonia fue identificada por MALDI-TOF MS como Abiotrophia defectiva, por lo que se cambió la terapia antimicrobiana a ampicilina intravenosa $2 \mathrm{~g}$, cuatro veces al día, más gentamicina $1 \mathrm{mg} / \mathrm{kg}$, fraccionada cada $8 \mathrm{~h}^{4}$. Se realizó un ecocardiograma transesofágico que mostró una válvula aórtica bicúspide intensamente engrosada con una vegetación de $9 \mathrm{~mm}$ asociada a una cavidad de $1,5 \times 1,5 \mathrm{~cm}$ en el anillo aórtico posterior adyacente a fibrosa mitroaórtica, con flujo diastólico desde aorta ascendente con comunicación hacia el tracto de salida del ventrículo izquierdo, sugiriendo un absceso drenado/ pseudoaneurisma (Figura 1).

Con estos elementos se decidió una cirugía con reemplazo valvular. Los hallazgos intraoperatorios mostraron tejido con aspecto inflamatorio, con presencia de múltiples vegetaciones sobre la válvula aórtica (Figura 2 y 3 ). Se realizó una valvectomía y descalcificación del anillo. Se objetivó a nivel anular en región de la valva no coronaria, una cavidad correspondiente a un absceso drenado espontáneamente. Se realizó curetaje y lavado profuso. Se colocó una prótesis mecánica St Jude $\mathrm{N}^{\circ} 21$. Se envió la válvula a cultivo, sin obtener desarrollo microbiológico probablemente porque la paciente se encontraba con tratamiento antibacteriano al momento de la cirugía.

Debido a una insuficiencia renal secundaria a fármacos se cambió se cambió ampicilina y gentamicina por vancomicina. Se utilizó dosis de $1 \mathrm{~g}$, fraccionada cada $12 \mathrm{~h}$. Se manejaron niveles plasmáticos adecuados de vancomicina en nadir $(15,4 \mathrm{ug} / \mathrm{dl})$, sin alteración de la función renal. La paciente completó tratamiento antimicrobiano por un total de cuatro semanas, con una evolución clínica favorable.

\section{Discusión}

La endocarditis infecciosa es una infección poco frecuente, pero con una importante morbilidad y mortalidad. El diagnóstico certero y rápido es clave para una evolución favorable. Existen algunos microorganismos poco comunes que se asocian a endocarditis. Ciertas bacterias requieren medios especiales o condiciones del medio enriquecidas para la identificación definitiva de las mismas.

Presentamos un caso de endocarditis provocada por una bacteria poco frecuente, $A$. defectiva diagnosticada por MALDI-TOF MS. Abiotrophia spp. corresponden a cocáceas grampositivas inmóviles, que al microscopio se presentan en pares o cadenas en medios líquidos en condiciones nutricionalmente adecuadas. La identificación convencional por métodos bioquímicos demanda entre 24 y $48 \mathrm{~h}$.

MALDI-TOF MS es una espectrometría de masas

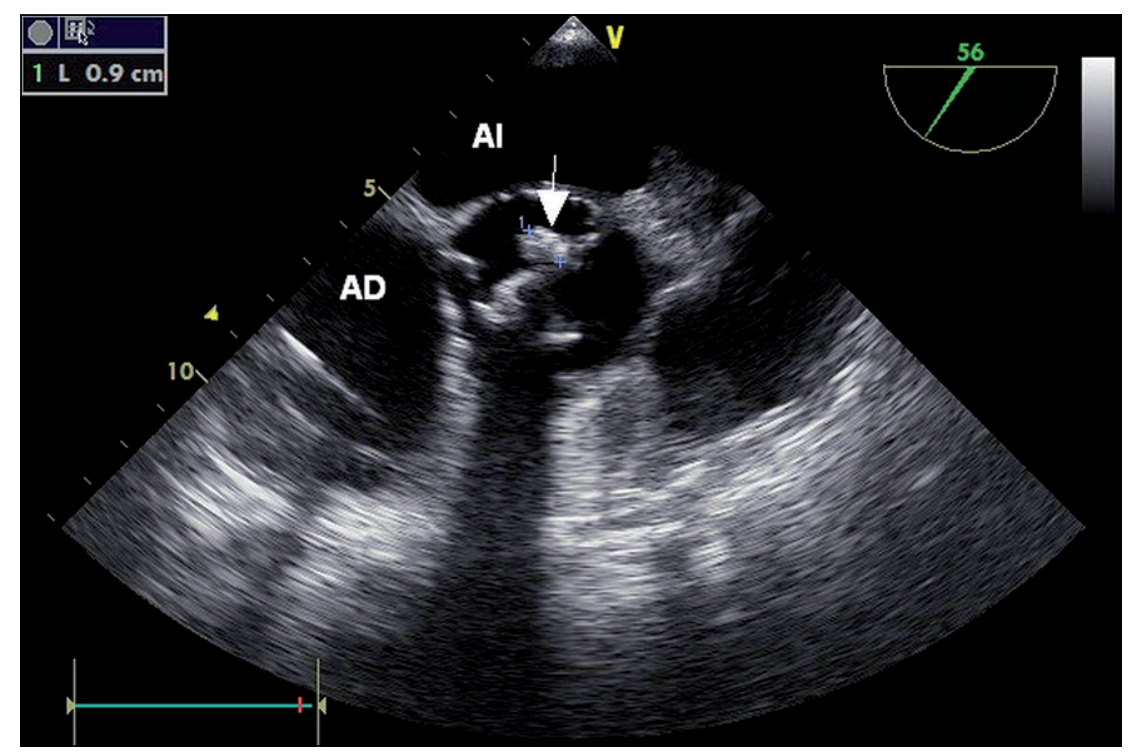

Figura 1. Ecocardiografia transfesofagica multiplanar de aorta obtenida desde la parte media del esófago a 56 . Al (aurícula izquierda), AD (aurícula derecha). Flecha: vegetación de $9 \mathrm{~mm}$ adherida a la valvula aórtica bicúspide.

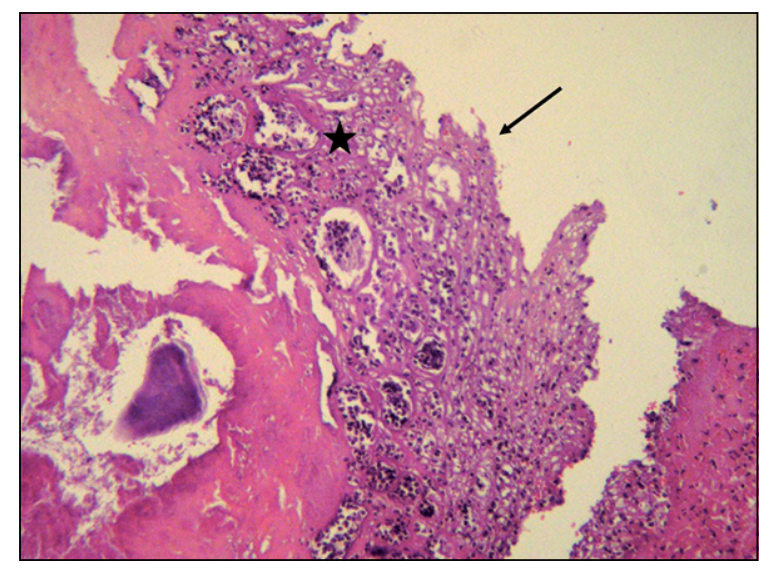

Figura 2. Corte histológico de válvula aórtica. Campo 10x con presencia de una estructura sobre-elevada e irregular, constituidas por una trama fibrinoleucocitaria, con polimorfonucleares neutrófilos (estrella), vinculables a vegetación valvular (flecha).

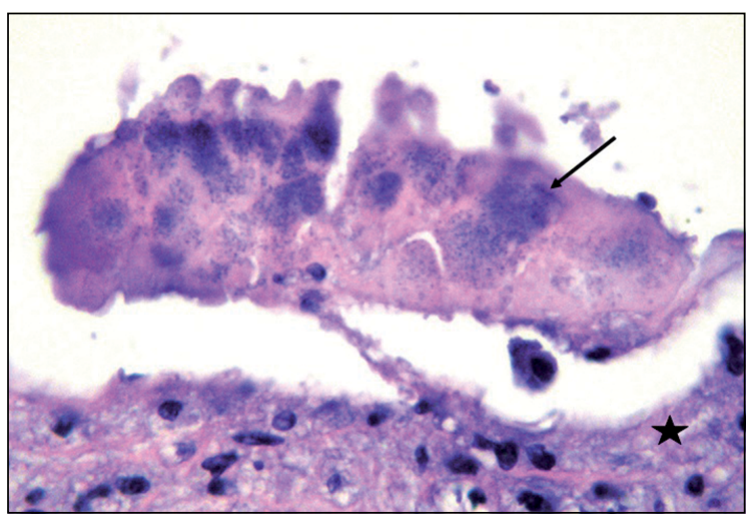

Figura 3. Sección con tinción con hematoxilina-eosina, en campo 40 x. Colonia de bacterias cocoides (flecha) en superficie valvular (estrella). 
que usa ionización con una matriz que se combina con la muestra a analizar (colonias bacterianas a partir de medios sólidos) para su análisis. Se basa en perfiles proteicos característicos de cada microorganismo, lo que permite identificar bacterias por comparación de los espectros con una base de datos. Con el uso de esta tecnología se logra acortar el proceso de diagnóstico microbiológico a $24 \mathrm{~h}^{8}$.

El uso de MALDI-TOF MS provee una identificación rápida y específica de las especies que componen el género Abiotrophia y permite diferenciarlo de los géneros afines ${ }^{9}$.

Los pacientes con endocarditis por Abiotrophia spp. deben ser tratados con un régimen similar a los que se recomienda para endocarditis por Enterococcus spp. Para el tratamiento específico de la endocarditis por Abiotrophia spp. se recomienda utilizar ampicilina intravenosa asociado a gentamicina durante cuatro a seis semanas, y en caso de falla terapéutica o alergia a antimicrobianos betalactámicos, el uso de vancomicina como monoterapia $\mathrm{o}$ asociada a gentamicina ${ }^{10}$.

En conclusión, se presenta un caso de endocarditis infecciosa por un patógeno de difícil crecimiento e infrecuente. Existen escasos reportes de endocarditis por $A$. defectiva en Argentina y Latinoamérica. El diagnóstico microbiológico mediante espectrometría de masa (MALDI-TOF MS) es rápido y confiable. La identificación certera de microorganismos permite la terapia adecuada de la endocarditis infecciosa.

\section{Resumen}

Se presenta el caso clínico de una mujer de 63 años de edad con antecedentes de una aorta bicúspide y estenosis aórtica grave, con una insuficiencia cardíaca descompensada. La paciente tuvo un síndrome febril con hemocultivos positivos para Abiotrophia defectiva. Se constató por un ecocardiograma transesofágico la presencia de un absceso paravalvular, por lo cual se realizó un reemplazo valvular en forma exitosa. Recibió terapia antimicrobiana intravenosa con vancomicina, con buena respuesta terapéutica. Se discute la utilización del MALDI-TOF MS como un método rápido y específico para el diagnóstico microbiológico.

\section{Referencias bibliográficas}

1.- Collins M D, Lawson P A. The genus Abiotrophia (Kawamura et al.) is not monophyletic: proposal of Granulicatella gen. nov., Granulicatella adiacens comb. nov., Granulicatella elegans comb. nov. and Granulicatella balaenopterae comb. nov. Int J Syst Evol Microbiol 2000; 50: 365-9.

2.- Frenkel A, Hirsch W. Spontaneous development of $\mathrm{L}$ forms of streptococci requiring secretions of other bacteria or sulphydryl compounds for normal growth. Nature 1961; 191: 728-30.

3.- Ruoff K L. Miscellaneous catalase-negative, gram-positive cocci: emerging opportunists. J Clin Microbiol 2002; 40: 1129-33.

4.- Brouqui P, Raoult D. Endocarditis due to rare and fastidious bacteria. Clin Microbiol Rev 2001; 14: 177-207.

5.- Greub G. MALDI-TOF mass spectrometry: the quantum leap. Clin Microbiol Infect 2010; 16: 1603.

6.- Ratcliffe P, Fang H, Thidholm E, Boräng S, Westling K, Özenci V. Comparison of MALDI-TOF MS and VITEK 2 system for laboratory diagnosis of Granulicatella and Abiotrophia species causing invasive infections. Diagn Microbiol Infect Dis 2013; 77: 216-9.

7.- Porte L, Zamorano J, Pavez D, Monckeberg G, Varela C, González P, et al. Endocarditis por Abiotrophia defectiva en paciente adulto. Rev Chilena Infectol 2004; 21: 151-5.

8.- Clark A, Kaleta E, Arora A, Wolk D. Matrixassisted laser desorption ionization-time of flight mass spectrometry: a fundamental shift in the routine practice of clinical microbiology. Clin Microbiol Rev 2013; 26: 547-603.

9.- Christensen J, Dargis R, Hammer M, Justesen U, Nielsen X, Kemp M, et al. Matrix-assisted laser desorption ionizationtime of flight mass spectrometry analysis of gram-positive, catalase-negative cocci not belonging to the Streptococcus or Enterococcus genus and benefits of database extension. J Clin Microbiol 2012; 50: 1787-91.

10.- Baddour L M, Wilson W R, Bayer A S, Fowler V G Jr, Tleyjeh I M, Rybak M J, et al. Infective endocarditis in adults: diagnosis, antimicrobial therapy, and management of complications: a scientific statement for healthcare professionals from the American Heart Association. Circulation 2015; 132: 1435-86. 